ARTICLE

DOI: $10.1038 / s 41467-018-06609-z$

\title{
Bacterial coinfection restrains antiviral CD8 T-cell response via LPS-induced inhibitory NK cells
}

Tobias Straub1, Marina A. Freudenberg 2,3 , Ulrike Schleicher ${ }^{4,5}$, Christian Bogdan ${ }^{4,5}$, Georg Gasteiger ${ }^{6,7} \&$ Hanspeter Pircher ${ }^{1}$

Infection of specific pathogen-free mice with lymphocytic choriomeningitis virus (LCMV) is a widely used model to study antiviral T-cell immunity. Infections in the real world, however, are often accompanied by coinfections with unrelated pathogens. Here we show that in mice, systemic coinfection with E. coli suppresses the LCMV-specific cytotoxic T-lymphocyte (CTL) response and virus elimination in a NK cell- and TLR2/4-dependent manner. Soluble TLR4 ligand LPS also induces NK cell-mediated negative CTL regulation during LCMV infection. NK cells in LPS-treated mice suppress clonal expansion of LCMV-specific CTLs by a NKG2D- or NCR1-independent but perforin-dependent mechanism. These results suggest a TLR4mediated immunoregulatory role of NK cells during viral-bacterial coinfections.

\footnotetext{
${ }^{1}$ Institute for Immunology, Medical Center - University of Freiburg, Faculty of Medicine, University of Freiburg, 79104 Freiburg, Germany. ${ }^{2}$ Institute for Biology III, Faculty of Biology, University of Freiburg, 79104 Freiburg, Germany. ${ }^{3}$ Department of Pneumology, Medical Center - University of Freiburg, Faculty of Medicine, University of Freiburg, 79104 Freiburg, Germany. ${ }^{4}$ Mikrobiologisches Institut - Klinische Mikrobiologie, Immunologie und Hygiene, Universitätsklinikum Erlangen, Friedrich Alexander-Universität (FAU) Erlangen-Nürnberg, 91054 Erlangen, Germany. ${ }^{5}$ Medical Immunology Campus Erlangen, FAU Erlangen-Nürnberg, 91054 Erlangen, Germany. ${ }^{6}$ Institute of Systems Immunology, University of Wuerzburg, 97078 Wuerzburg, Germany. ${ }^{7}$ Institute for Medical Microbiology and Hygiene, University of Freiburg Medical Center, 79104 Freiburg, Germany. Correspondence and requests for materials should be addressed to H.P. (email: hanspeter.pircher@uniklinik-freiburg.de)
} 
nfection of mice with lymphocytic choriomeningitis virus (LCMV) is widely used to study innate and adaptive immune responses. Depending on viral strain and virus dose, LCMV induces either an acute infection, characterized by a potent virusspecific cytotoxic CD8 ${ }^{+}$T-lymphocyte $(\mathrm{CTL})$ response followed by rapid virus clearance, or a chronic infection with $\mathrm{T}$ cell exhaustion and virus persistence. In most cases, clean laboratory mice kept under specific pathogen-free (SPF) conditions have been used for these studies. However, viral infections in real life may be accompanied by coinfections with unrelated pathogens that have the potential to modulate anti-viral immune responses ${ }^{1}$. The impact of a LCMV infection on a coinfection with bacterial pathogens has been analyzed in a number of studies. These data show that the LCMV infection can aggravate secondary infections with certain bacteria but may also protect against Gram-positive pathogens ${ }^{2-4}$. Enhanced susceptibility of LCMV-infected mice to LPS treatment has also been reported ${ }^{5-7}$. However, the reverse scenario, i.e., the effect of a bacterial coinfection on LCMVspecific T-cell immunity, has so far only been analyzed in a polymicrobial sepsis model ${ }^{8}$. These experiments showed that sepsis induced by cecal-ligation and puncture strongly impaired subsequent induction of a LCMV-specific CTL response ${ }^{9-12}$. Mechanistically, these findings have been explained by apoptosisinduced loss of antigen presenting cells ${ }^{12}$, decrease in LCMVspecific precursor $\mathrm{T}$-cells ${ }^{10}$, alterations in memory CD8 T-cell function $^{11}$ or exacerbation of T-cell exhaustion ${ }^{9}$.

NK cells are well-known for their potent antiviral and antitumoral activity but it is also evident that they function as important regulators of adaptive immunity during viral infections. In the murine cytomegalovirus (MCMV) infection model, NK cell-depletion prior to infection has been shown to improve $\mathrm{T}$-cell responses and consequently virus elimination ${ }^{13-15}$. For infection with LCMV, which is not primarily controlled by NK cells, it was demonstrated that NK cells suppress antiviral immunity by killing activated CD4 and CD8 T-cells ${ }^{16-18}$. Accordingly, ablation of NK cells before or during chronic LCMV infection led to a stronger $\mathrm{T}$-cell response and more efficient virus clearance ${ }^{19,20}$. By suppressing the CD4 T-cell response, NK cell regulatory activity also effects immune memory and $\mathrm{B}$ cell immunity during LCMV infection ${ }^{21,22}$. Importantly, these regulatory activities of NK cells during LCMV infection were only observed when high $\left(>10^{4} \mathrm{pfu}\right)$ infectious doses were used for inoculation. In low dose (200 pfu) infection settings, NK cell depletion did not improve the LCMV-specific CTL response and virus clearance ${ }^{23-25}$.

NK cells activated during bacterial infections were found to contribute to bacteria elimination but also to disease pathogenesis $^{26}$. NK cell activation in these infections can occur both directly by sensing of bacteria through pattern recognition receptors and indirectly via bacterial stimulation of dendritic cells or macrophages ${ }^{27}$. In case of $E$. coli infection and its major pathogen-associated molecular pattern LPS, it was demonstrated that NK cell activation is facilitated via IL-2, IL-18 and IFN- $B$ produced by dendritic cells ${ }^{28}$. In view of the reported regulatory activity of NK cells, we hypothesized that bacterial coinfection may result in enhanced NK cell regulatory activity. Indeed, we here demonstrate that NK cells in LPS-treated mice suppress clonal expansion of LCMV-specific CTLs by a NKG2Dindependent or NCR1-independent but perforin-dependent mechanism. These results suggest a TLR4-mediated immunoregulatory role of NK cells during viral-bacterial coinfections.

\section{Results}

E. coli coinfection interferes with LCMV control. To determine whether a bacterial coinfection can interfere with LCMV-specific
CTL immunity, C57BL/6 (B6) mice were infected with a low dose (200 pfu) of LCMV (strain WE) followed by inoculation with $5 \times$ $10^{5} \mathrm{cfu}$ of $E$. coli one day later. At day 8 post-infection (p.i.), the LCMV-specific CTL response was analyzed by MHC class I tetramer staining and by assessing viral titers. Without coinfection, the mice generated a robust virus-specific CTL response and decreased viral titer to low levels. Interestingly, coinfection with E. coli significantly reduced the LCMV-specific CTL response and strongly impaired virus elimination in spleen and liver. Most strikingly, antibody-mediated depletion of $\mathrm{NK}$ cells almost completely restored the LCMV-specific CTL response and virus clearance in E. coli coinfected mice (Fig. 1a-c).

To determine whether the negative effect of $E$. coli coinfection on anti-LCMV CD8 T cell immunity was mediated by bacterial cell wall components such as LPS or peptidoglycans, TLR2/4deficient mice were used. In striking contrast to wild-type (wt) mice, E. coli coinfection $\left(2 \times 10^{6} \mathrm{cfu}\right)$ of TLR2/4-deficient mice did neither inhibit the LCMV-specific CTL response nor impair viral clearance. In addition, depletion of $\mathrm{NK}$ cells before coinfection did not significantly improve the antiviral CTL response in these mice (Fig. 1d, e). Taken together, these data suggest that cell wall components released during $E$. coli infection enabled NK cells to interfere with induction of LCMV-specific CTL and virus clearance.

TLR ligands inhibit the anti-LCMV CTL response. To provide direct evidence that TLR4 triggering was able to interfere with the induction of LCMV-specific T cell immunity, purified LPS $(1 \mu \mathrm{g})$ was injected into B6 mice that had been infected with LCMV one day earlier. At day 8 p.i., the LCMV-specific CTL response and viral titers were analyzed. Similar to the E. coli coinfection, LPS injection also strongly reduced the LCMV-specific CTL response and prevented rapid virus clearance. Importantly, NK cell depletion again reverted the negative effects of LPS treatment on induction of the LCMV-specific CTL response and virus elimination (Fig. 2a, c). Absolute numbers of splenocytes were comparable in all experimental groups indicating that gp33- and np396-tetramer ${ }^{+}$CTL were de- or increased not only in relative but also in absolute numbers (Fig. 2b). Similar to LPS, injection of TLR3 ligand poly(I:C) or TLR9 ligand CpG oligodeoxynucleotides (ODN) also markedly suppressed the virus-specific T-cell response and virus clearance through a NK cell-dependent mechanism (Supplementary Fig. 1). LPS treatment did not suppress the anti-LCMV CTL response in TLR2/4-deficient mice. However, injection of poly(I:C) resulted in a decreased CTL response and impaired virus control, demonstrating that these mice were still responsive to NK cell activating signals (Supplementary Fig. 2). Taken together, these data show that TLR ligands when present at an early time point after LCMV infection strongly interfered with rapid viral clearance by a NK celldependent mechanism.

NK cell-mediated inhibition of CTL is perforin-dependent. To analyze the effect of LPS on the induction of LCMV-specific CTL at early time points, we used an adoptive transfer system with LCMV gp33-specific CD8 T-cells from P14 TCR transgenic mice. A tracer population of P14 T-cells (Thy $1.1^{+}$) was transferred into B6 recipient mice followed by LCMV infection and LPS or PBS injection at day 1 p.i.. At day 4 after infection, P14 T-cell frequencies in spleens of infected mice were slightly increased compared to non-infected controls. This initial expansion was, however, not affected by the LPS treatment (Fig. 3a, left). Likewise, the rate of BrdU incorporation in P14 T cells was not influenced by LPS administration (Fig. 3a, right). These data indicate that LPS-treatment in the context of a LCMV infection 
a

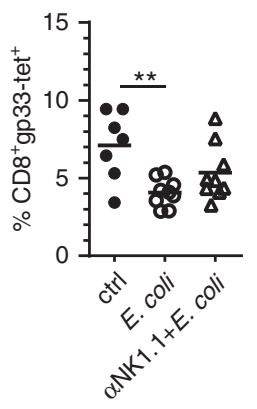

d

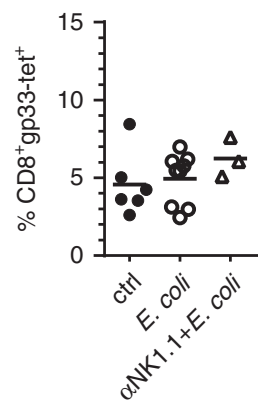

b

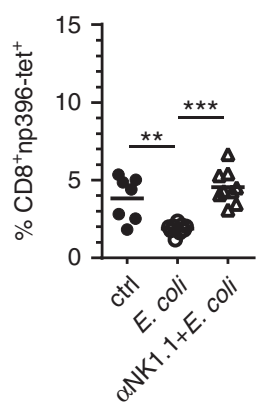

TLR2/4-/-

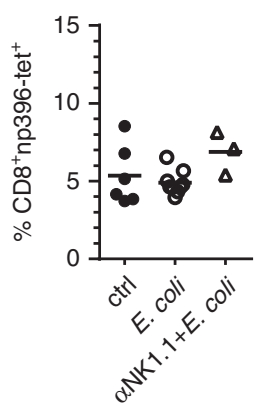

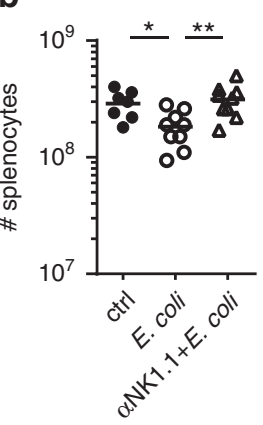

C
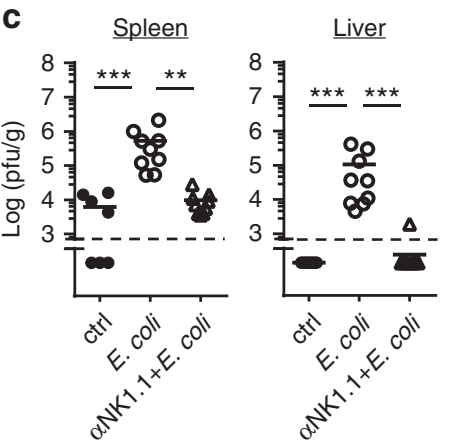

e

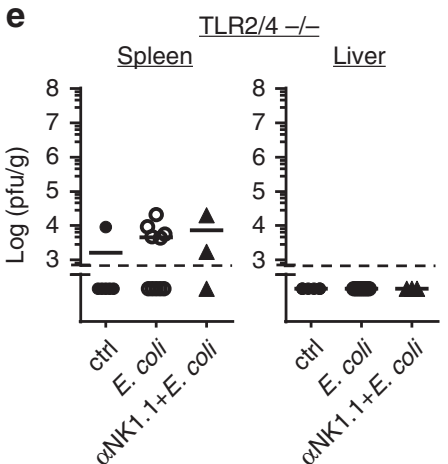

Fig. 1 E. coli coinfection leads to NK cell-mediated impairment of the anti-LCMV CTL response. NK cell-depleted and non-depleted B6 (a-c) or TLR2/4deficient mice (d, e) were infected with LCMV. One day later, they were coinfected with E. coli or received sterile LB medium as control (ctrl) and were analyzed at day 8 after LCMV infection. a, d gp33- and np396-tetramer ${ }^{+}$(tet) CD8 T cells in percent of spleen cells. b Absolute numbers (\#) of splenocytes. c, e Viral titers in spleen and liver. Data of individual mice $(n=7-9(\mathbf{a}-\mathbf{c}), n=3-9(\mathbf{d}, \mathbf{e}))$ from 1-3 independent experiments with 2-4 mice per group are shown; horizontal bars represent the mean values. Dashed lines indicate detection limits. ${ }^{\star} p<0.05,{ }^{\star \star} p<0.01,{ }^{\star \star \star} p<0.001 ;$ one-way ANOVA with Tukey-Kramer post-test (a, b), Mann-Whitney (d, e), or Kruskal-Wallis Test with Dunn's post-test (c)
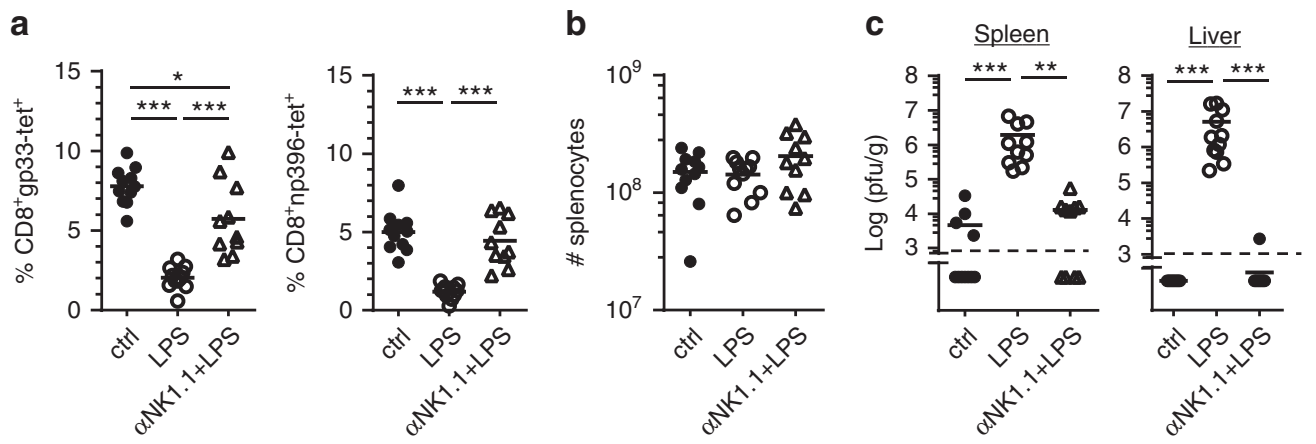

Fig. 2 LPS treatment leads to NK cell-mediated impairment of the anti-LCMV CTL response. NK cell-depleted and non-depleted B6 mice were infected with LCMV. After one day of infection, they received LPS or PBS as a control (ctrl). Mice were analyzed at day 8 after LCMV infection. a gp33- and np396tetramer ${ }^{+}$(tet) CD8 T cells in percent of spleen cells. $\mathbf{b}$ Absolute numbers (\#) of splenocytes. $\mathbf{c}$ Viral titers in spleen and liver. Data of individual mice $(n=$ 11) from 4 to 5 independent experiments with 2-3 mice per group are shown; horizontal bars represent the mean values. Dashed lines indicate detection limits. ${ }^{\star} p<0.05,{ }^{\star \star} p<0.01,{ }^{\star \star \star} p<0.001$; one-way ANOVA with Tukey-Kramer post-test $(\mathbf{a}, \mathbf{b})$ or Kruskal-Wallis Test with Dunn's post-test (c)

did not interfere with the initial priming of the CTL response. In sharp contrast to this, one day later (day 5 p.i.), frequencies and absolute numbers of clonally expanded P14 T-cells were considerably lower in LPS-treated mice compared to controls (Fig. 3b). As in the polyclonal setting, the LPS-induced decrease in P14 T-cell expansion was prevented by NK cell depletion (Fig. 3c). To confirm the importance of NK cells for the decreased expansion of P14 T cells after LCMV infection and LPS treatment, IL-15-deficient mice were used as recipients of P14 T cells. These mice almost completely lack NK cells but mount a fully functional CTL immune response after LCMV infection ${ }^{29}$. In contrast to wt recipient mice, LPS injection did not lower the
LCMV-induced expansion of P14 T cells in IL-15-deficient mice (Fig. 3d).

Regulation of T-cells by NK cells frequently operates through cell-mediated lysis via perforin $16,17,30$. To determine whether the decrease in expansion of P14 T-cells by LPS was perforindependent, perforin-deficient mice were used as recipients of P14 $\mathrm{T}$ cells. Unlike to wt recipients, LPS did not decrease the LCMVinduced expansion of P14 T-cells in hosts lacking perforin (Fig. 3e). For the LCMV clone 13 infection model, it was postulated that NK cells negatively regulate $\mathrm{T}$ cell priming by cellmediated lysis of antigen presenting cells (APC) ${ }^{19}$. The unaltered initial P14 T cell expansion until day 4 p.i. (Fig. 3a), however, 

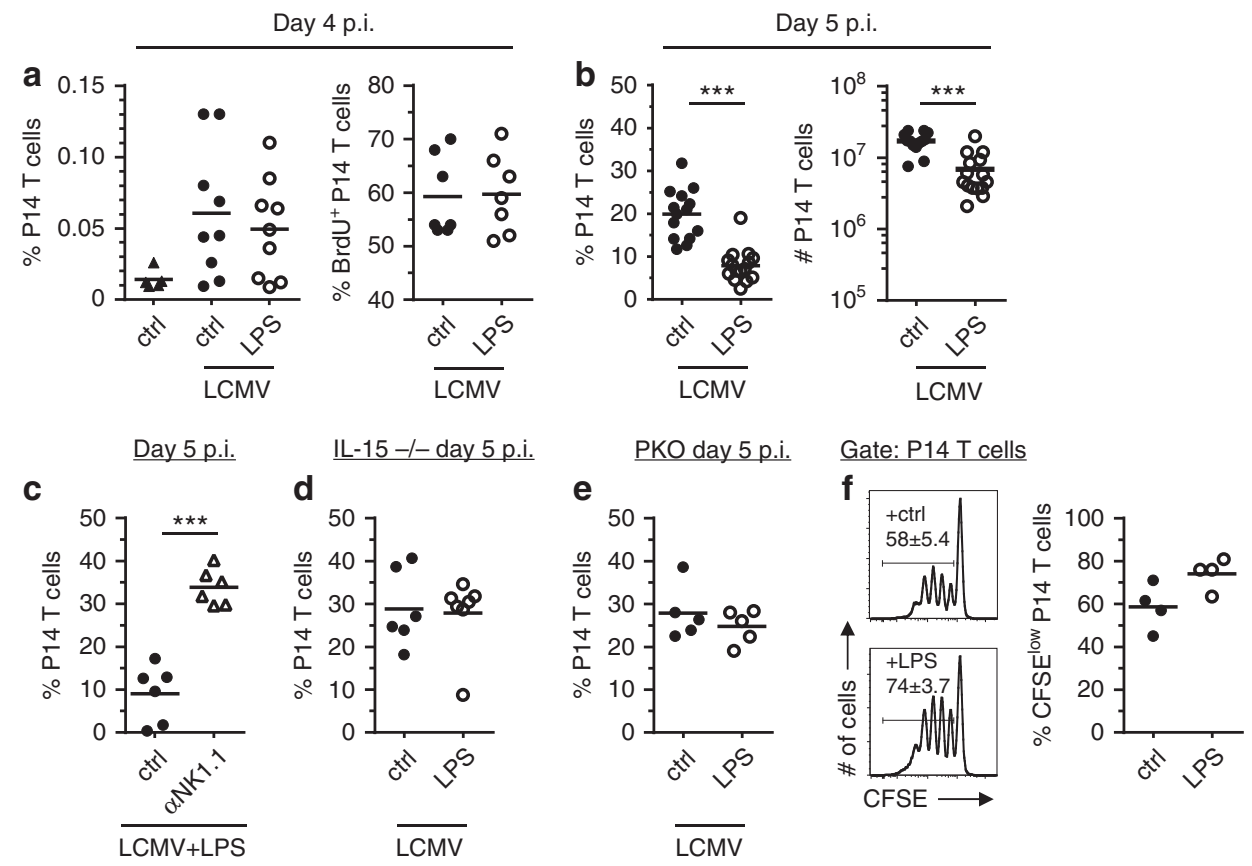

Fig. 3 LPS-activated NK cells inhibit clonal expansion of LCMV-specific P14 CD8 T-cells. P14 T-cells were transferred into the indicated recipients which were subsequently infected with LCMV. After one day, they received LPS or PBS as a control (ctrl). a Percentages P14 T-cells of splenocytes (left) and BrdU + cells of splenic P14 T cells (right) at day 4 p.i. or day 4.5 p.i. respectively. For BrdU labeling, mice were injected with BrdU and sacrificed 20 min later. Data are pooled from 2 to 4 independent experiments with 1-3 mice per group $(n=5-9)$. b Percentages P14 T cells of splenocytes (left) and absolute numbers (\#) of P14 T-cells in spleen (right) at day 5 p.i.. Data are pooled from seven independent experiments with 1-3 mice per group ( $n=13-15$ ). c Percentages P14 T cells of splenocytes in NK cell-depleted and non-depleted B6 at day 5 p.i.. d, e Percentages P14 T cells of splenocytes in IL-15 $\%$ - and perforin $^{-/-}$mice at day 5 p.i.. Data are pooled from 2 to 3 independent experiments with 2-3 mice per group $(n=5-7)$. f CFSE-labeled P14 T cells were stimulated with splenic APCs from LCMV-infected B6 mice (day 4 p.i.) that had been injected with LPS ( $1 \mu \mathrm{g}$ ) or PBS at day 1 p.i.. After 3 days, cell division of P14 T cells was analyzed by dye dilution. Representative histograms are shown, numbers in histograms indicate mean values \pm SEM; dots represent mean values of duplicates. Data are pooled from two independent experiments $(n=4)$ with 1-3 mice per group. ${ }^{\star \star \star} p<0.001 ;$ Mann-Whitney Test

already suggested that APC capacity is unlikely to be affected in our setting. To further determine whether LPS injection interfered with priming of $\mathrm{T}$ cells, we used splenic APC from LCMV-infected mice (day 4 p.i.) to stimulate proliferation of naïve P14 T-cells. As depicted in Fig. 3f, APC isolated from LPStreated mice showed an even slightly increased capacity to stimulate P14 T cell proliferation in vitro. Taken together, these data indicate that NK cells activated directly or indirectly by LPS during an ongoing LCMV infection negatively regulated proliferating LCMV-specific $\mathrm{CD}^{+}$T-cells by a perforindependent mechanism.

LPS promotes NK cell accumulation after LCMV infection. NK cells become activated during LCMV infection ${ }^{31}$ but their numbers hardly increase (Fig. 4a). Interestingly, LPS given at day 1 after LCMV infection led to significantly increased NK cell frequencies and numbers in the spleen at day 4 p.i.. Likewise, NK cell numbers in liver and lungs were also considerably increased in LCMV/LPS- compared to LCMV/PBS-treated mice (Supplementary Fig. 3). Without infection, LPS treatment did not increase splenic NK cell frequencies and numbers. The cytolytic activity of LCMV/LPS-activated NK cells was tested in ${ }^{51} \mathrm{Cr}$ release-assays using NK cell-sensitive YAC-1 target cells. Splenocytes from LCMV/LPS-treated mice showed a 3 to 5-fold increase in lytic activity against YAC-1 cells compared to splenocytes from LCMV-infected mice without LPS treatment (Fig. 4b, left). This difference was primarily due to the increased NK cell frequency since lytic activity of NK cells on a per cell basis was only slightly increased by LPS injection (Fig. 4b, right). NK cells from LPS-treated LCMV-infected mice also displayed a more mature phenotype with smaller $\mathrm{CD} 11 \mathrm{~b}^{-} \mathrm{CD} 27^{+}$but larger
$\mathrm{CD}_{11 b^{+}} \mathrm{CD}_{27}{ }^{+}$subsets and increased KLRG1 expression (Fig. 4c).

To test whether direct TLR signaling in NK cells was required for LPS-induced cell proliferation during LCMV infection, CFSElabeled NK cells from TLR2/4-deficient or wt mice were transferred into wt mice, followed by LCMV infection and LPS injection. After 4 days, cell division of the transferred NK cells was analyzed by CFSE dye dilution. LPS injection into LCMVinfected recipients significantly increased NK cell division as evident by an increased portion of CFSE ${ }^{\text {low }}$ NK cells. This increase was also evident in NK cells lacking TLR2/4 (Fig. 4d). Thus, LPS given in the context of a LCMV infection promoted NK cell proliferation indirectly by a NK cell-extrinsic pathway.

Role of IL-15 in negative T-cell regulation. IL-15 is an important cytokine for NK cell survival and activation that is also induced by LPS $^{32,33}$. To block IL-15 signaling, we used a monoclonal antibody (mAb) that specifically targets the IL-15/IL15R complex. Without LPS treatment, blocking IL-15/IL-15R did not affect induction of LCMV-specific CTL and virus clearance (Fig. 5d-f) confirming previous findings that IL-15 is not required for effector T-cell induction ${ }^{29}$. Anti-IL-15/IL-15R mAb treatment, however, limited the increase of NK cells after LPS injection in the context of a LCMV infection (Fig. 5b). Nonetheless, anti-IL-15/IL-15R mAb treatment did not restore the LCMVspecific CTL response and virus elimination after LPS injection (Fig. 5a, c). Thus, IL-15 signaling was dispensable for the LPStriggered negative $\mathrm{T}$-cell regulation by NK cells. In addition, these data imply that the increased NK cell numbers observed after LPS injection were not a prerequisite for their suppressive effect on the LCMV-specific CTL response. 

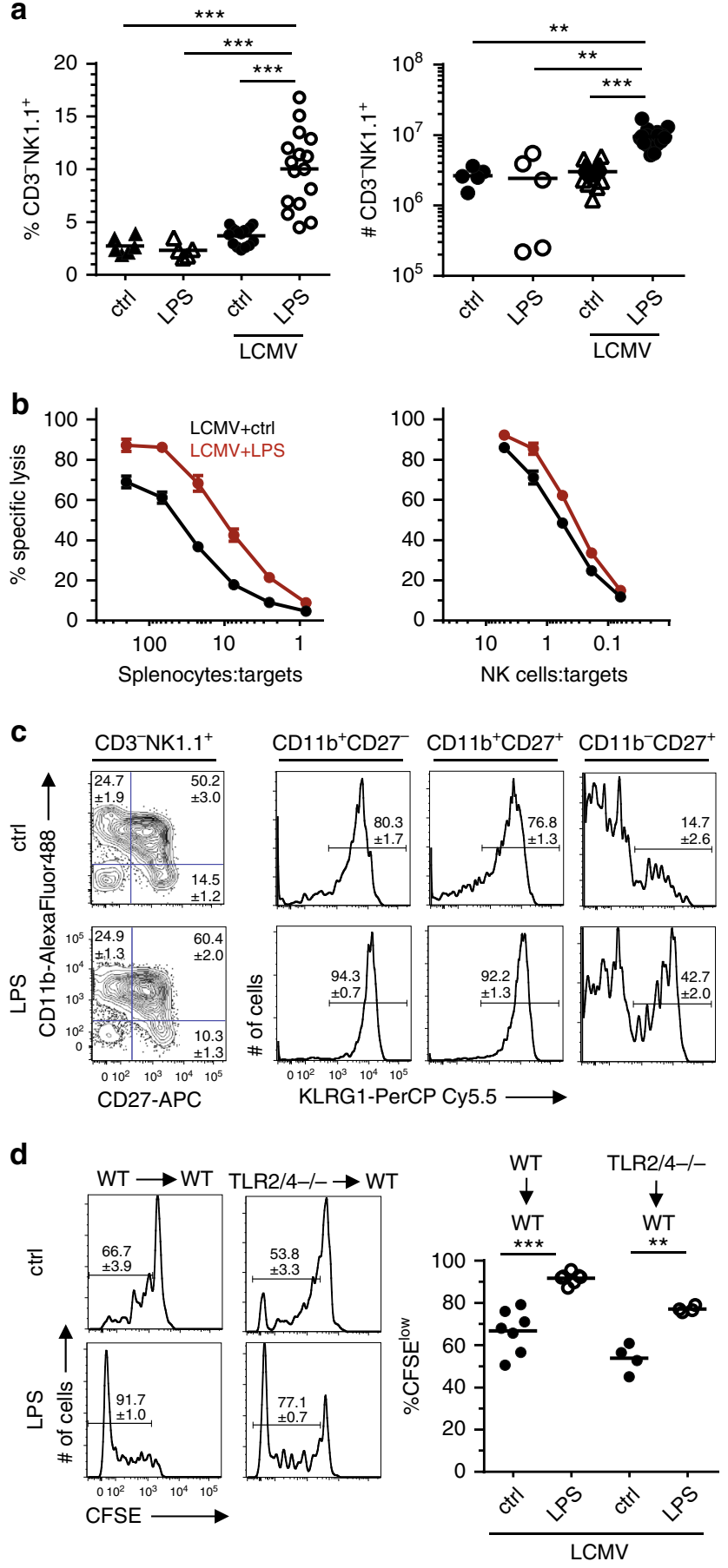

IFN- $\beta$, IL-2 and IL-18 have also been shown to be necessary and sufficient for NK cell activation following exposure to E. coli or LPS administration; induction of NK cell cytotoxic activity required IFN- $\beta$ and IL-15 but not IL- 2 or IL- $18^{28}$. In our setting, however, IFN- $\beta$ and IL-18 were dispensable for the suppressive effect of LPS-activated NK cells on the LCMV-specific T-cell response (Supplementary Fig. 4A to D). Also the combined deficiency of IL-18 and IL-12 did not prevent suppression of LCMV-specific T-cell immunity by LPS-activated NK cells (Supplementary Fig. 4E to F).

NK cell-mediated inhibition is independent of NKG2D or NCR1. NKG2D and NCR1 have both been reported to be
Fig. 4 LPS promotes NK cell accumulation after LCMV infection. B6 mice were infected with LCMV, injected with LPS or PBS one day later and analyzed at day 4 p.i.. a Percentage and absolute numbers (\#) of NK cells $\left(\mathrm{CD}^{-}{ }^{\left.-N K 1.1^{+}\right)}\right.$in spleens. Data are pooled from five independent experiments ( $n=5-16)$ with 2-4 mice per group. b Specific lysis of YAC-1 target cells by NK cells. Results are displayed as total splenocyte-to target cell-ratio (left, $n=15$ ) or as NK cell-to-target cell-ratio (right, $n=7$ ). Data are pooled from 2 to 6 independent experiments with 2-4 mice per group. Dots represent mean values, error bars the SEM. c Expression of CD11b, CD27 and KLRG1, gated at the indicated NK cell subsets. Pooled data from five independent experiments $(n=11)$ with 2-4 mice per group and representative contour plots and histograms are shown. Numbers indicate means \pm SEM. d CFSE-labeled wild-type (wt) or TLR2/4 /- NK cells were transferred into $B 6$ recipient mice. One day later, recipient mice were infected with LCMV and another day later injected with LPS or PBS. At day 4 p.i., cell division of the transferred NK cells (CD45.2 $\left.2^{+} \mathrm{CD}^{-} \mathrm{NK} 1.1^{+}\right)$in spleen was analyzed by CFSE dye dilution. Representative histograms are shown, numbers indicate mean values \pm SEM; dots represent values of individual mice. The positions of the gates were determined by the utmost right peak of the CFSE dilution histograms which represents undivided cells. The gates include all cells with lower fluorescence intensity when compared to the undivided CSFEhigh cells. Data are derived from five independent experiments with 1-3 mice per group (for wt NK cells, $n=7$ ) and two independent experiments with two mice per group (for TLR2/4 $/-\mathrm{NK}$ cells, $n=4) .{ }^{\star} p<0.05,{ }^{\star *} p<0.01,{ }^{\star *} p<0.001$; ANOVA with Tukey-Kramer post-test (a, left); Kruskal-Wallis with Dunn's post-Test (a, right); unpaired $t$-test with Welch-correction (d)

responsible for killing of activated LCMV- and MCMV-specific CD8 T-cells by NK cells ${ }^{16,23,30}$. The importance of NKG2D in our model system was tested using NKG2D-deficient mice. The data show that LPS given in the context of a LCMV infection also strongly decreased the LCMV-specific CTL response and virus elimination in the absence of NKG2D (Fig. 6a-d). To assess the role of the activating NK cell receptor NCR1, we utilized two different mouse strains. First, similar to Crouse et al. ${ }^{23}$, we used NKp $46^{\text {icre/icre }}$ mice which exhibit a strongly impaired NCR1 expression ${ }^{34}$. In these mice we found that LPS injection still suppressed the LCMV-specific CTL response and impaired virus elimination (Fig. 6e, g, h). However, NK cell frequencies in LCMV-infected NKp $4 \sigma^{\text {icre/icre }}$ mice were considerably decreased when compared to wt mice (Fig. 6f). Therefore, we tested an additional recently described mouse line termed B6.CD45.1$\mathrm{Ncr}{ }^{14 \mathrm{R}}$ that lacks NCR1 cell surface expression due to a point mutation in the NCR1 signal peptide ${ }^{35}$. In these mice, NK cell frequencies were not affected by the lack of NCR1 expression (Fig. 6j). Nonetheless, LPS injection also significantly suppressed the anti-LCMV CTL response and increased viral burdens in the absence of NCR1 (Fig. 6i, j, l). Together, these results demonstrate that NKG2D and NCR1 were dispensable for LPS-induced NK cell-mediated suppression of the anti-LCMV CD8 T cell immune response.

\section{Discussion}

Several studies have previously shown that NK cells are able to kill antigen-specific T-cells after LCMV infection. Importantly, this type of negative regulation of T-cells by NK cells in LCMV infection is only observed after inoculation with high virus doses $\left(>10^{4} \mathrm{pfu}\right)^{16,17,19}$. When mice were infected with low doses $(200$ pfu) of LCMV-WE, as it was done in the present study, NK celldepletion did not significantly improve the LCMV-specific CTL response and virus clearance ${ }^{23-25}$. Using this low-dose infection setting, we now demonstrate that TLR2/4 ligands generated during a coinfection with $E$. coli caused a NK cell-mediated 
a

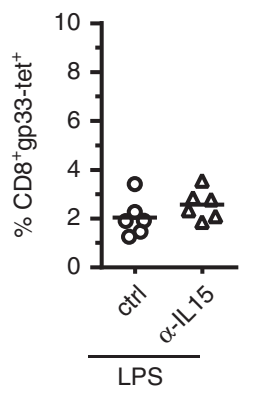

d

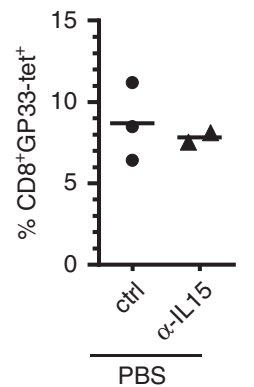

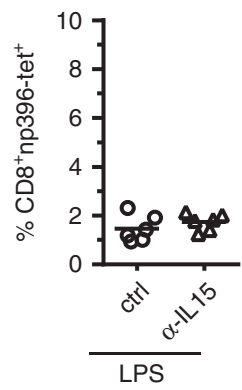

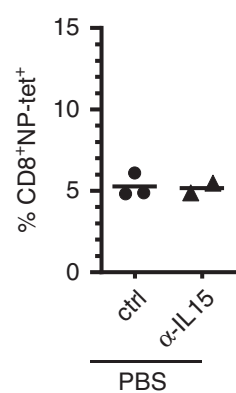

b

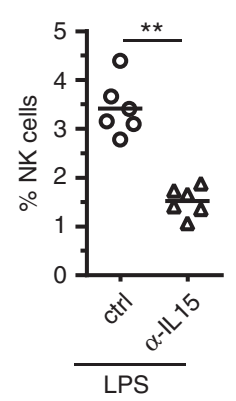

e

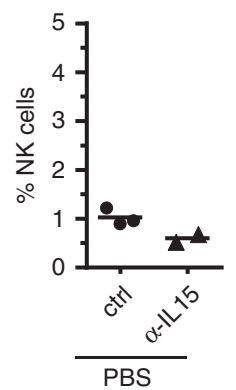

C
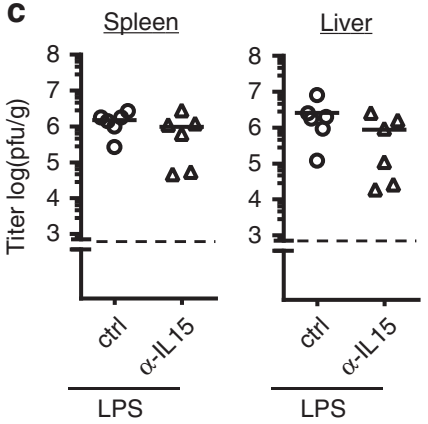

f

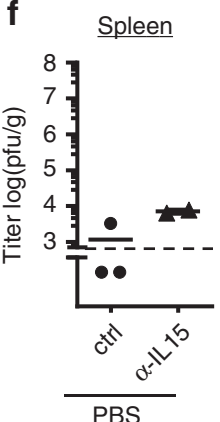

Liver

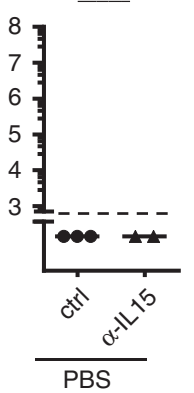

Fig. 5 IL-15 blockade inhibits LPS-induced NK cell accumulation but not negative T-cell regulation. B6 mice were infected with LCMV and received LPS (a-c) or PBS (d-f) at day 1 p.i.. As indicated, groups of mice were additionally treated at day 1 and day 3 p.i. with mAb specific for IL-15/IL-15R complex. All mice were analyzed at day 8 after LCMV infection. a, d gp33- and np396-tetramer ${ }^{+}$(tet) CD8 ${ }^{+} \mathrm{T}^{-}$cells in percent of spleen cells in the indicated groups. b, e Percent NK cells (CD3-NK1.1 ${ }^{+}$) of spleen cells. (c, f) Viral titers in spleen and liver. Data of individual mice from (a-c) two independent experiments with three mice per group $(n=6)$ and $\mathbf{d}-\mathbf{f}$ one experiment with $2-3$ mice per group $(n=2-3)$ are shown; horizontal bars represent the mean values. Dashed lines indicate detection limits. ${ }^{\star \star} p<0.01$, Mann-Whitney Test

suppression of the anti-viral CTL response and thereby prevented rapid virus control. Similarly, injection of the soluble TLR ligands LPS, poly(I:C) or CpG ODN also impaired the LCMV-specific CTL response and virus elimination through a NK cell-dependent mechanism. These findings reveal a hitherto unknown mechanism by which bacterial infections incapacitate a central antiviral effector pathway.

NK cells in LPS-treated mice suppressed the expansion of LCMV-specific P14 CD8 T-cells by a perforin-dependent mechanism. This suppression took place between day 4 and day 5 p.i., when the activated P14 cells underwent an extensive proliferative burst. Besides activated T-cells, LCMV antigen presenting cells (APC) may also serve as targets for NK cells ${ }^{19}$. However, LPS injection did not affect the capacity of ex vivo isolated splenic APCs from LCMV-infected mice to stimulate P14 $\mathrm{T}$-cell proliferation in vitro. In addition, LPS treatment did not impair initial in vivo expansion of P14 T-cells up to day 4 p.i. and did not affect BrdU incorporation in P14 T-cells at day 4 p.i.. Hence, these data suggest that upon TLR ligand treatment NK cells kill proliferating virus-specific CD8 T-cells. In line with previous studies showing that CD4 T-cells can also serve as targets of NK cell regulatory activity ${ }^{16,17}$, we observed that LPStreatment decreased clonal expansion of LCMV-specific SMARTA CD4 ${ }^{+}$T-cells after LCMV infection as well (Supplementary Fig. 5). Since control of low dose LCMV infection is predominantly dependent on $\mathrm{CD}^{+}{ }^{+} \mathrm{T}$-cells, it is, however, unlikely that impaired virus elimination after LPS injection was primarily due to the missing LCMV-specific $\mathrm{CD}^{+}{ }^{+} \mathrm{T}$-cell response.

Dendritic cells are able to sense pathogens through pattern recognition receptors and, in turn, activate NK cells ${ }^{36-38}$. IL-15 induced by type I IFN-receptor signaling is further important for TLR4-triggered NK cell activation ${ }^{28,37}$. Antibody blockade of IL15-signaling significantly impaired the LPS-induced accumulation of NK cells during LCMV infection in our system. Nonetheless, this treatment failed to prevent their suppressive effect on the LCMV-specific CTL response. This indicates that the sole increase in NK cell numbers as observed in LCMV-infected mice after LPS injection was not a prerequisite for the suppression. Remarkably, IL-12, IL-18, and IFN- $\beta$ were also dispensable. This suggests that the cytokine requirement for the inhibitory effect of NK cells on the LCMV-specific CTL response after TLR4 stimulation exhibits a considerable redundancy. This could be due to the two potent immunological stimuli, LCMV infection and LPS injection, used here.

Several receptors have been reported to be important for killing of activated $\mathrm{CD}^{+}{ }^{+}$or $\mathrm{CD} 8^{+}$T-cells by NK cells. A crucial role of NKG2D was shown in two studies performed in the LCMV and in the MCMV model systems ${ }^{16,30}$. In contrast, Waggoner et al. ${ }^{18}$ showed that absence of $2 \mathrm{~B} 4$ promoted NK cell-mediated killing of LCMV-activated $\mathrm{CD} 8^{+} \mathrm{T}$-cells but found no evidence for the involvement of NKG2D. Similar to Waggoner et al. ${ }^{18}$ and Crouse et al. ${ }^{23}$, we also did not observe a role of NKG2D in the negative regulation of LCMV-specific $\mathrm{CD}^{+}{ }^{-} \mathrm{T}$-cells by $\mathrm{NK}$ cells. The reason for this discrepancy is unknown and remains to be solved.

Absence of the type I IFN receptor (IFNAR) has been shown to render activated LCMV-specific CD8 ${ }^{+}$T-cells more susceptible to NK cell killing ${ }^{23,25}$. In addition, adoptive transfer experiments with IFNAR-deficient P14 T-cells into Ncr1 $1^{\text {icre/icre }}$ mice revealed an essential role of NCR1 in this process. In our experiments with

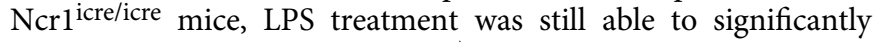
decrease the LCMV-specific CD8 ${ }^{+}$T-cell response. However, the extent of this decrease as well as the increase in viral titers after LPS treatment was less pronounced in $N K p 46^{\text {icre/icre }}$ mice than in wt mice. This could be due to the lower NK cell frequencies in NKp46 icre/icre mice or non-defined strain differences. Importantly, LPS-treatment also significantly lowered the 
a

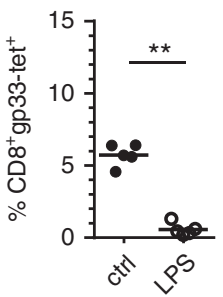

e

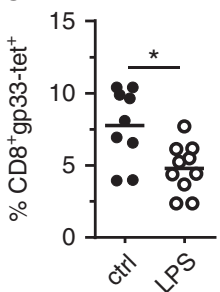

i

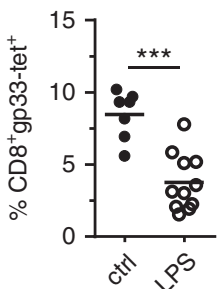

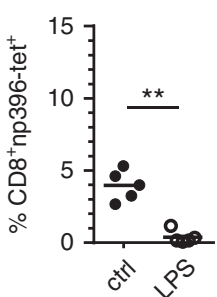

b

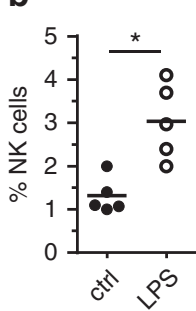

f
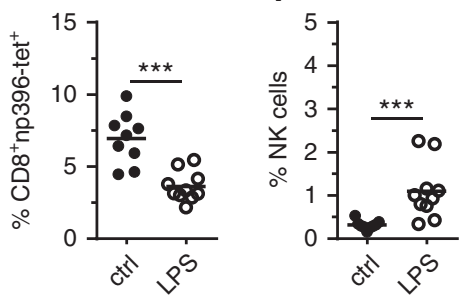

g
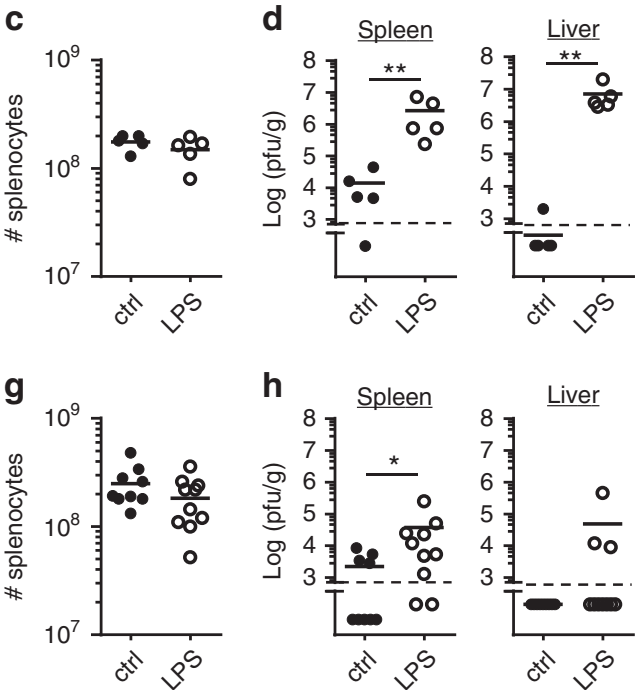

h
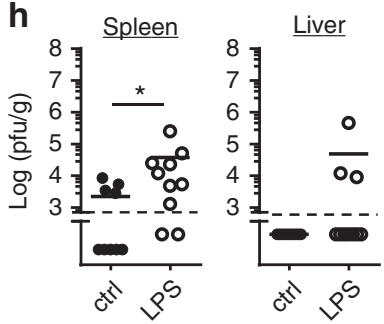

j
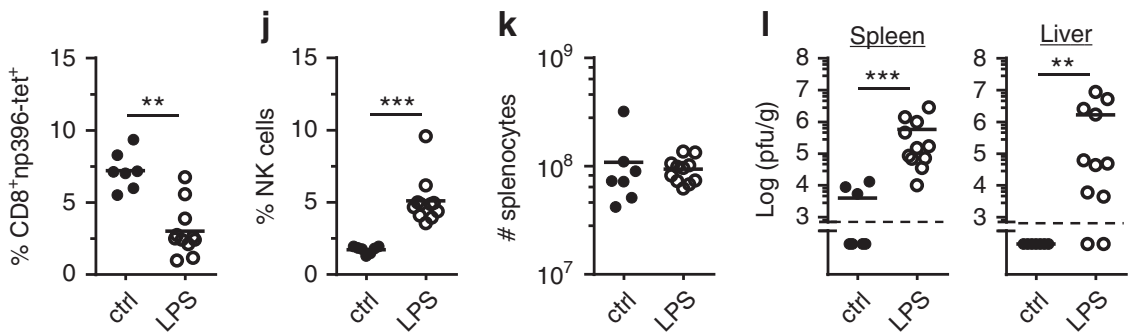

Fig. 6 Deficiency in NCR1 or NKG2D does not prevent LPS-induced negative T cell regulation. NKG2D-deficient $(\mathbf{a}-\mathbf{d}, n=5), N K p 46$ icre/icre $(\mathbf{e}-\mathbf{h}, n=9-10)$ and B6.CD45.1-Ncr C14R mice (i-I, $n=8-11)$ were infected with LCMV. One day later, they received LPS or PBS (ctrl). Mice were analyzed at day 8 after LCMV infection. a, e, i gp33- and np396-tetramer ${ }^{+}$(tet) CD8 T cells in percent of spleen cells. $\mathbf{b}, \mathbf{f}, \mathbf{j}$ Percent NK cells (CD3-NK1.1 ${ }^{+}$) of spleen cells. $\mathbf{c}, \mathbf{g}, \mathbf{k}$ Absolute numbers (\#) of spleen cells. $\mathbf{d}, \mathbf{h}, \mathbf{I}$ Viral titers in spleen and liver. Data of individual mice from 2 to 3 independent experiments with 2-6 mice per group are shown; horizontal bars represent the mean values. Dashed lines indicate detection limits. ${ }^{\star} p<0.05,{ }^{\star \star} p<0.01,{ }^{\star \star \star} p<0.001$;

Mann-Whitney test

LCMV-specific CTL response and increased viral burdens in B6. CD45.1-Ncr ${ }^{\mathrm{C} 14 \mathrm{R}}$ mice that lack NCR1 cell surface expression but contain normal NK cell numbers ${ }^{35}$. Thus, these data indicate that NCR1 was not involved in the interaction of LPS-activated NK cells with activated LCMV-specific CD8 ${ }^{+} \mathrm{T}$ cells.

$\mathrm{T}$-cells protect themselves from NK cell killing by expression of ligands for inhibitory $\mathrm{NK}$ cell receptors or by suppression of ligands for activating NK cell receptors ${ }^{18,23,39}$. For instance, type I IFNs are known to induce expression of MHC class I and Ib molecules that function as ligands for NK cell inhibitory receptors ${ }^{25,40}$. We observed that IFN- $\alpha$ and IFN- $\beta$ levels in serum of LCMV-infected mice were roughly two-fold reduced at $24 \mathrm{~h}$ after LPS injection as compared to control mice. However, this decrease did not alter $\mathrm{H}-2 \mathrm{~K}^{\mathrm{b}}, \mathrm{H}-2 \mathrm{D}^{\mathrm{b}}$ and $\mathrm{Qa}-1 \mathrm{~b}$ expression levels on LCMV-specific P14 CD8 T-cells isolated from NK celldepleted hosts at day 5 p.i. (Supplementary Fig. 6). Thus, negative $\mathrm{T}$-cell regulation by $\mathrm{NK}$ cells in LPS-treated mice occurred despite high expression of MHC class I and Ib molecules.

While the impact of viral infections on bacterial coinfections has been extensively studied in the past ${ }^{41-44}$, the reverse regulation as described here has been poorly recognized to date. There are a few reports that previously demonstrated an effect of coinfection on LCMV-specific T-cell immunity. Polymicrobial sepsis analyzed in a cecal-ligation and puncture model has been shown to impair LCMV-specific T-cell immunity by various mechanism including loss of APC function, decrease in precursor $\mathrm{T}$-cell frequencies and alterations in T-cell functions ${ }^{9-12}$. Mice co-infected with LCMV and Schistosoma mansoni further show enhanced early LCMV replication and impaired viral clearance in the liver most likely due to a decreased type I IFN response in this $\operatorname{organ}^{45}$. Similarly, decreased type I IFN production that limits expansion of LCMV-specific CTL has been observed in mice coinfected with Ectromelia virus and $\mathrm{LCMV}^{46}$. Finally, coinfection of mice with Pichinde virus led to a decreased number of LCMVspecific CTL but increased variability in immunodominance that can be rationalized by the competition between two simultaneous immune responses 47 .

In conclusion, the present study sheds light on an additional aspect in the complex network of viral-bacterial coinfections. In particular, it links TLR stimulation by bacterial pathogens to NK cell-mediated suppression of virus-specific T cells, which has not been reported previously. The results have important implications for our understanding of the immune defense and mechanisms of evasion when the organism is simultaneously exposed to two different pathogens.

\section{Methods}

Mice. C57BL/6 (B6) mice were obtained from Janvier (Le Genest St-Isle, France). B6-Ly5.2/Cr $\left(\mathrm{CD} 45.1^{+}\right)$, IL-15-deficient ${ }^{48}$, IL-18-deficient ${ }^{49}$, perforin-deficient ${ }^{50}$, TLR2/4-deficient ${ }^{51}$, NKG2D-deficient ${ }^{52}, N K p 46^{\text {icre/icre53 }}$, IFN- $\beta^{\text {luc/luc54, }}$ P14 TCR $\mathrm{tg}^{55}$, SMARTA TCR $\mathrm{tg}^{56}$ and B6.CD45.1-Ncr ${ }^{\mathrm{C} 14 \mathrm{R} 35}$ mice were bred locally. P14 chimeric mice were generated by adoptive transfer (i.v.) of $1 \times 10^{5}$ splenic P14 $\mathrm{TCR}^{+}$tg T-cells into B6 mice. Mice were bred and kept in our animal facility under specific pathogen-free conditions. Animal care and use was approved by the Regierungspräsidium Freiburg. All experiments were performed in accordance with the German law for animal protection.

Infections and treatments. Mice were infected i.v. with 200 pfu LCMV-WE. For coinfection experiments, B6 and TLR2/4-deficient mice were infected one day later with $5 \times 10^{5}$ or $2 \times 10^{6}$ cfu E. coli EH100, respectively, in Lysogeny Broth (LB) medium $(200 \mu$ l, i.v. $)$. E. coli were grown in $\mathrm{LB}$ medium and frozen at $-80^{\circ} \mathrm{C}$ in glycerol stocks. Before infection, E. coli were regrown in LB medium until an 
optical densitiy (OD $600 \mathrm{~nm}$ ) of 0.4 was reached. Infection dose of bacteria was calculated from the linear relationship between turbidity and the number of cfu grown after $24-48 \mathrm{~h}$ at $37^{\circ} \mathrm{C}$. Control mice received sterile LB medium. For TLRligand treatment, mice received one day after LCMV infection $1 \mu \mathrm{g}$ LPS derived from E. coli O111:B4 (Sigma-Aldrich), $50 \mu \mathrm{g}$ poly(I:C) (Enzo) or $50 \mu \mathrm{g}$ CpG ODN 1668 (InvivoGen) in $200 \mu \mathrm{l}$ PBS via the intravenous route; controls received PBS only. NK cells were depleted by injection of $300 \mu \mathrm{g}$ and $200 \mu \mathrm{g}$ purified anti-NK1.1 $\mathrm{mAb}$ (clone PK136, BioXcell) at day 2 and day 1, respectively, before LCMV infection. In the P14 T-cell transfer experiments (Fig. 3c), control (ctrl) mice received mouse IgG2a isotype control mAb (clone C1.18.4, BioXcell). Since treatment with isotype control $\mathrm{mAb}$ had no effect on the anti-LCMV CTL response and virus clearance (Supplementary Fig. 7), isotype control antibodies were omitted for the NK cell-depletion experiments without P14 T-cell transfers (Fig. 1 and Fig. 2). For blocking IL-15 signaling, mice were treated at day 1 and 3 after infection (i.p.) with anti-IL-15/IL-15R a chain complex antibody $(50 \mu$ g, clone GRW15PLZ, eBioscience) or isotype control mAb (clone HRPN, BioXcell) or left untreated. For blocking IL-12, anti-IL-12 p40 antibody ( $200 \mu \mathrm{g}$, clone C17.8, BioXcell) was given (i.p.) at day 0 and at day 1 and 3 after infection.

Flow cytometry. For isolation of lymphocytes, spleens were meshed through a metal strainer. Livers were meshed through a cell strainer (Greiner) and lymphocytes purified using a Percoll (Sigma) gradient. Lungs were digested using Collagenase II (Roche, $140 \mathrm{U} \mathrm{ml}^{-1}$ ) and DNaseI (Sigma, $10 \mu \mathrm{g} \mathrm{ml}^{-1}$ ) and meshed through a cell strainer. The following $\mathrm{mAbs}$ were purchased from BioLegend (BL), eBioscience (eB) or Miltenyi Biotec (MB): anti-CD8 a (clone 53.6.7, cat. \# 100723 (BL), diluted 1:100), anti-Thy1.1 (HIS51, \#14-0900-81 (eB), 1:1000), anti-CD3ع (145-2C11, \#100312, 1:100), anti-CD11b (M1/70, \#101217 (BL), 1:100), anti-CD27 (LG.7F9, \#124211 (BL), 1:500), anti-KLRG1 (2F1, \#138418 (BL), 1:150), antiCD45.1 (A20, \#110724 (BL), 1:200), anti-NK1.1 (PK136, \#108714, 1:500), anti-CD4

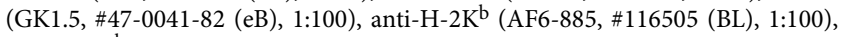
anti-H-2D ${ }^{\mathrm{b}}$ (KH95, \#111508 (BL), 1:50), anti-Qa-1B (6A8.6F10.1 A6, \#13-105-048 (MB), 1:10). Zombie NIR dye (BioLegend) or DAPI (Sigma) was used for dead cell exclusion. To detect virus-specific $\mathrm{CD} 8^{+} \mathrm{T}$ cells, lymphocytes were stained with $\mathrm{D}^{\mathrm{b}} \mathrm{GP} 33$ and $\mathrm{D}^{\mathrm{b}} \mathrm{NP} 396$ tetramers ${ }^{57}$ (produced in-house). Staining was performed for at least $20 \mathrm{~min}$ at $4{ }^{\circ} \mathrm{C}$. Samples were measured on a LSR Fortessa or Canto II cytometer (both BD Biosciences) and data were analyzed with FlowJo software 8.8.7 (Tree Star).

${ }^{51}$ Chromium-release assay. Cytolytic activity of NK cells was determined by a standard ${ }^{51}$ Chromium-release assay. In brief, serial 1:3-dilutions of effector cells were mixed in 96-well round bottom plates with YAC-1 target cells (obtained from Dr. Rolf Zinkernagel, Zürich) that had been loaded with ${ }^{51}$ Chromium (PerkinElmer) for $2 \mathrm{~h}$ at $37^{\circ} \mathrm{C}$. Total splenocytes or enriched NK cells (MojoSort NK cell isolation kit, BioLegend) were used as effector cells. After incubation at $37^{\circ} \mathrm{C}$ for 5 $\mathrm{h}$, radioactivity in the supernatant was measured using a gamma-counter. Duplicate wells were assayed for each effector-target ratio and percentages of specific lysis were calculated.

In vitro T-cell stimulation assay. To test antigen presenting cell (APC) function, spleens from LPS-treated and control-treated LCMV-infected mice at day 4 p.i. were digested with collagenase II (Roche, $2000 \mathrm{U} / \mathrm{ml}$ ) for $30 \mathrm{~min}$ at $37^{\circ} \mathrm{C}$ and subsequently minced through a metal strainer. Afterwards, $2 \times 10^{5}$ splenocytes depleted of Thyl. $2^{+}$and $\mathrm{B} 220^{+}$cells by positive selection (Dynabeads Magnetic Separation, ThermoFisher) were co-cultured with $2 \times 10^{5}$ CFSE-labeled enriched (mouse CD8 T-cell isolation kit, Miltenyi Biotec) P14 TCR ${ }^{+} \mathrm{T}$ cells in in 96-well plates for 3 days. Afterwards, cell division of P14 T-cells was analyzed by dye dilution.

NK cell proliferation in vivo. NK cells were enriched from CFSE-labeled splenocytes of indicated donor mice using mouse CD49b-Microbeads (Miltenyi Biotec) or the MagniSort Mouse NK cell isolation kit (eBioscience). $3 \times 10^{5}$ to $8 \times 10^{5}$ enriched CFSE-labeled NK cells were transferred one day prior to infection into indicated mice expressing a different CD45 isoform.

Viral titers. Viral titers were determined by standard focus-forming assay ${ }^{58}$. In brief, organs were homogenized using a FastPrep-24 (MPBiomedicals). Serial 1:10dilutions of tissue homogenate were plated on MC57G fibrosarcoma cells (obtained from Dr. Rolf Zinkernagel, Zürich) in 24-well plates and after $4 \mathrm{~h}$ incubation at $37^{\circ} \mathrm{C}$, an overlay containing $1 \%$ methylcellulose was added. After another incubation for $40 \mathrm{~h}$ at $37^{\circ} \mathrm{C}$, supernatant was discarded and cells were fixed with $4 \%$ formaldehyde in PBS, followed by permeabilization using $0.5 \%$ Triton X-100 in PBS, blocking with $10 \%$ FCS in PBS and staining with anti-LCMV NP mAb (clone VL-4, made in-house) and horse radish peroxidase-conjugated polyclonal goatanti-rat IgG antibody (Jackson ImmunoResearch) as secondary antibody. Foci were detected by incubation with SIGMAFAST OPD (Merck).

Serum type I IFN concentrations. Mouse sera were obtained using BD Microtainer SST tubes. IFN- $\alpha$ concentration in the sera was determined using the
VeriKine Mouse Interferon Alpha ELISA Kit (pbl), IFN- $\beta$ concentration was determined using the BD Cytometric Bead Array Mouse inflammation Kit, both according to the manufacturers instructions.

Statistics. Statistical differences between two groups were determined using unpaired two-tailed $t$-test or Mann-Whitney test depending on whether data demonstrated Gaussian distribution or not. When data were sampled from Gaussian distribution but had different standard deviations, unpaired two-tailed $t$ test with Welsh correction was used. Statistical differences between more than two groups were determined using two-tailed one-way ANOVA with Tukey-Kramer post-test or two-tailed Kruskal-Wallis test with Dunn's post-test depending on whether data demonstrated Gaussian distribution or not. All tests were performed using the GraphPad InStat software.

\section{Data availability}

All relevant data are available from the authors upon request.

Received: 11 April 2018 Accepted: 12 September 2018

Published online: 08 October 2018

\section{References}

1. Stelekati, E. \& Wherry, E. J. Chronic bystander infections and immunity to unrelated antigens. Cell Host. Microbe 12, 458-469 (2012).

2. Gumenscheimer, M. et al. Stage of primary infection with lymphocytic choriomeningitis virus determines predisposition or resistance of mice to secondary bacterial infections. Med. Microbiol. Immunol. 196, 79-88 (2007).

3. Merches, K. et al. Virus-induced type i interferon deteriorates control of systemic Pseudomonas Aeruginosa infection. Cell Physiol. Biochem. 36, 2379-2392 (2015)

4. Navarini, A. A. et al. Increased susceptibility to bacterial superinfection as a consequence of innate antiviral responses. Proc. Natl Acad. Sci. USA 103, 15535-15539 (2006)

5. Doughty, L., Nguyen, K., Durbin, J. \& Biron, C. A role for IFN-alpha beta in virus infection-induced sensitization to endotoxin. J. Immunol. 166, 2658-2664 (2001).

6. Nansen, A., Christensen, J. P., Marker, O. \& Thomsen, A. R. Sensitization to lipopolysaccharide in mice with asymptomatic viral infection: role of $\mathrm{T}$ celldependent production of interferon-gamma. J. Infect. Dis. 176, 151-157 (1997).

7. Nansen, A. \& Randrup Thomsen, A. Viral infection causes rapid sensitization to lipopolysaccharide: central role of IFN-alpha beta. J. Immunol. 166, 982-988 (2001).

8. Jensen, I. J., Sjaastad, F. V., Griffith, T. S. \& Badovinac, V. P. Sepsis-induced T cell immunoparalysis: the Ins and outs of impaired $\mathrm{T}$ cell immunity. $J$. Immunol. 200, 1543-1553 (2018).

9. Condotta, S. A., Khan, S. H., Rai, D., Griffith, T. S. \& Badovinac, V. P. Polymicrobial sepsis increases susceptibility to chronic viral infection and exacerbates CD8+ T Cell Exhaustion. J. Immunol. 195, 116-125 (2015).

10. Condotta, S. A., Rai, D., James, B. R., Griffith, T. S. \& Badovinac, V. P. Sustained and incomplete recovery of naive CD8 $+\mathrm{T}$ cell precursors after sepsis contributes to impaired CD8+ T cell responses to infection. J. Immunol. 190, 1991-2000 (2013).

11. Duong, S. et al. Polymicrobial sepsis alters antigen-dependent and -independent memory CD8 T cell functions. J. Immunol. 192, 3618-3625 (2014).

12. Strother, R. K. et al. Polymicrobial sepsis diminishes dendritic cell numbers and function directly contributing to impaired primary CD8 $\mathrm{T}$ cell responses in vivo. J. Immunol. 197, 4301-4311 (2016).

13. Andrews, D. M. et al. Innate immunity defines the capacity of antiviral $\mathrm{T}$ cells to limit persistent infection. J. Exp. Med. 207, 1333-1343 (2010).

14. Schuster, I. S. et al. TRAIL + NK cells control CD4+ T cell responses during chronic viral infection to limit autoimmunity. Immunity 41, 646-656 (2014).

15. Su, H. C. et al. NK cell functions restrain $\mathrm{T}$ cell responses during viral infections. Eur. J. Immunol. 31, 3048-3055 (2001).

16. Lang, P. A. et al. Natural killer cell activation enhances immune pathology and promotes chronic infection by limiting CD8(+) T-cell immunity. Proc. Natl Acad. Sci. USA 109, 1210-1215 (2012).

17. Waggoner, S. N., Cornberg, M., Selin, L. K. \& Welsh, R. M. Natural killer cells act as rheostats modulating antiviral T cells. Nature 481, 394-398 (2011).

18. Waggoner, S. N., Taniguchi, R. T., Mathew, P. A., Kumar, V. \& Welsh, R. M Absence of mouse 2B4 promotes NK cell-mediated killing of activated CD8+ $\mathrm{T}$ cells, leading to prolonged viral persistence and altered pathogenesis. J. Clin Invest. 120, 1925-1938 (2010). 
19. Cook, K. D. \& Whitmire, J. K. The depletion of NK cells prevents T cell exhaustion to efficiently control disseminating virus infection. J. Immunol. 190, 641-649 (2013).

20. Waggoner, S. N., Daniels, K. A. \& Welsh, R. M. Therapeutic depletion of natural killer cells controls persistent infection. J. Virol. 88, 1953-1960 (2014).

21. Cook, K. D., Kline, H. C. \& Whitmire, J. K. NK cells inhibit humoral immunity by reducing the abundance of CD4 $+\mathrm{T}$ follicular helper cells during a chronic virus infection. J. Leukoc. Biol. 98, 153-162 (2015).

22. Rydyznski, C. et al. Generation of cellular immune memory and B-cell immunity is impaired by natural killer cells. Nat. Commun. 6, 6375 (2015)

23. Crouse, J. et al. Type I interferons protect T cells against NK cell attack mediated by the activating receptor NCR1. Immunity 40, 961-973 (2014).

24. Ehl, S. et al. A comparison of efficacy and specificity of three NK depleting antibodies. J. Immunol. Methods 199, 149-153 (1996).

25. Xu, H. C. et al. Type I interferon protects antiviral CD8+ T cells from NK cell cytotoxicity. Immunity 40, 949-960 (2014).

26. Souza-Fonseca-Guimaraes, F., Adib-Conquy, M. \& Cavaillon, J. M. Natural killer (NK) cells in antibacterial innate immunity: angels or devils? Mol. Med. 18, 270-285 (2012).

27. Adib-Conquy, M., Scott-Algara, D., Cavaillon, J. M. \& Souza-FonsecaGuimaraes, F. TLR-mediated activation of NK cells and their role in bacterial/ viral immune responses in mammals. Immunol. Cell Biol. 92, 256-262 (2014).

28. Zanoni, I. et al. IL-15 cis presentation is required for optimal NK cell activation in lipopolysaccharide-mediated inflammatory conditions. Cell Rep. 4, 1235-1249 (2013).

29. Becker, T. C. et al. Interleukin 15 is required for proliferative renewal of virusspecific memory CD8 T cells. J. Exp. Med. 195, 1541-1548 (2002).

30. Soderquest, $\mathrm{K}$. et al. Cutting edge: CD8+ T cell priming in the absence of NK cells leads to enhanced memory responses. J. Immunol. 186, 3304-3308 (2011).

31. Welsh, R. M. Jr. Cytotoxic cells induced during lymphocytic choriomeningitis virus infection of mice. I. Characterization of natural killer cell induction. $J$. Exp. Med. 148, 163-181 (1978).

32. Carson, W. E. et al. Interleukin (IL) 15 is a novel cytokine that activates human natural killer cells via components of the IL-2 receptor. J. Exp. Med. 180, 1395-1403 (1994)

33. Doherty, T. M., Seder, R. A. \& Sher, A. Induction and regulation of IL-15 expression in murine macrophages. J. Immunol. 156, 735-741 (1996).

34. Narni-Mancinelli, E. et al. Tuning of natural killer cell reactivity by NKp46 and Helios calibrates T cell responses. Science 335, 344-348 (2012).

35. Jang Y. et al. Cutting edge: check your mice-a point mutation in the Ncrl locus identified in CD45.1 Congenic mice with consequences in mouse susceptibility to infection. J. Immunol. 200, 1982-1987 (2018).

36. Fernandez, N. C. et al. Dendritic cells directly trigger NK cell functions: crosstalk relevant in innate anti-tumor immune responses in vivo. Nat. Med. 5, 405-411 (1999).

37. Lucas, M., Schachterle, W., Oberle, K., Aichele, P. \& Diefenbach, A. Dendritic cells prime natural killer cells by trans-presenting interleukin 15. Immunity 26, 503-517 (2007).

38. Schleicher, U. et al. NK cell activation in visceral leishmaniasis requires TLR9, myeloid DCs, and IL-12, but is independent of plasmacytoid DCs. J. Exp. Med. 204, 893-906 (2007).

39. Ludigs, K. et al. NLRC5 shields T lymphocytes from NK-cell-mediated elimination under inflammatory conditions. Nat. Commun. 7, 10554 (2016).

40. $\mathrm{Xu}, \mathrm{H}$. C. et al. Lymphocytes negatively regulate NK cell activity via Qa-1b following viral infection. Cell Rep. 21, 2528-2540 (2017).

41. Bakaletz, L. O. Viral-bacterial co-infections in the respiratory tract. Curr. Opin. Microbiol. 35, 30-35 (2017).

42. Jamieson, A. M. et al. Role of tissue protection in lethal respiratory viralbacterial coinfection. Science 340, 1230-1234 (2013).

43. McCullers, J. A. The co-pathogenesis of influenza viruses with bacteria in the lung. Nat. Rev. Microbiol. 12, 252-262 (2014).

44. Melvin, J. A. \& Bomberger, J. M. Compromised defenses: exploitation of epithelial responses during viral-bacterial co-infection of the respiratory tract. PLoS. Pathog. 12, e1005797 (2016).

45. Edwards, M. J. et al. Reciprocal immunomodulation in a schistosome and hepatotropic virus coinfection model. J. Immunol. 175, 6275-6285 (2005).

46. McAfee, M. S., Huynh, T. P., Johnson, J. L., Jacobs, B. L. \& Blattman, J. N. Interaction between unrelated viruses during in vivo co-infection to limit pathology and immunity. Virology 484, 153-162 (2015).

47. Kenney, L. L. et al. Increased immune response variability during simultaneous viral coinfection leads to unpredictability in CD8 T cell immunity and pathogenesis. J. Virol. 89, 10786-10801 (2015).
48. Kennedy, M. K. et al. Reversible defects in natural killer and memory CD8 T cell lineages in interleukin 15-deficient mice. J. Exp. Med. 191, 771-780 (2000).

49. Takeda, K. et al. Defective NK cell activity and Th1 response in IL-18-deficient mice. Immunity 8, 383-390 (1998).

50. Kagi, D. et al. Cytotoxicity mediated by T cells and natural killer cells is greatly impaired in perforin-deficient mice. Nature 369, 31-37 (1994).

51. Lembo, A. et al. Differential contribution of Toll-like receptors 4 and 2 to the cytokine response to Salmonella enterica serovar Typhimurium and Staphylococcus aureus in mice. Infect. Immun. 71, 6058-6062 (2003).

52. Guerra, N. et al. NKG2D-deficient mice are defective in tumor surveillance in models of spontaneous malignancy. Immunity 28, 571-580 (2008).

53. Narni-Mancinelli, E. et al. Fate mapping analysis of lymphoid cells expressing the NKp46 cell surface receptor. Proc. Natl Acad. Sci. USA 108, 18324-18329 (2011).

54. Lienenklaus, S. et al. Novel reporter mouse reveals constitutive and inflammatory expression of IFN-beta in vivo. J. Immunol. 183, 3229-3236 (2009).

55. Pircher, H., Burki, K., Lang, R., Hengartner, H. \& Zinkernagel, R. M. Tolerance induction in double specific $\mathrm{T}$-cell receptor transgenic mice varies with antigen. Nature 342, 559-561 (1989).

56. Oxenius, A., Bachmann, M. F., Zinkernagel, R. M., Hengartner, H. \& Virusspecific, M. H. C. class II-restricted TCR-transgenic mice: effects on humoral and cellular immune responses after viral infection. Eur. J. Immunol. 28 390-400 (1998).

57. Altman J. D., Davis M. M. MHC-peptide tetramers to visualize antigenspecific T cells. Curr. Protoc. Immunol. 115, 1713 11-17 1344 (2016)

58. Battegay, M. et al. Quantification of lymphocytic choriomeningitis virus with an immunological focus assay in 24- or 96-well plates. J. Virol. Methods 33, 191-198 (1991).

\section{Acknowledgements}

The work was supported by the Deutsche Forschungsgemeinschaft DFG (SFB 1160/P3 to H.P. and SP1937 to G.G.). We thank Prof. Eric Vivier for providing NKp46 icre/icre mice and Dr. Peter Aichele for critical comments on the manuscript.

\section{Author contributions}

T.S. designed, planed and performed the experiments, analyzed data and wrote the paper M.A.F., U.S., C.B. and G.G. provided reagents or mice and were involved in data discussion and drafting the manuscript. H.P. initiated, organized and designed the study, wrote the paper and completed the manuscript.

\section{Additional information}

Supplementary Information accompanies this paper at https://doi.org/10.1038/s41467 018-06609-z.

Competing interests: The authors declare no competing interests.

Reprints and permission information is available online at http://npg.nature.com/ reprintsandpermissions/

Publisher's note: Springer Nature remains neutral with regard to jurisdictional claims in published maps and institutional affiliations.

Open Access This article is licensed under a Creative Common Attribution 4.0 International License, which permits use, sharing, adaptation, distribution and reproduction in any medium or format, as long as you give appropriate credit to the original author(s) and the source, provide a link to the Creative Commons license, and indicate if changes were made. The images or other third party material in this article are included in the article's Creative Commons license, unless indicated otherwise in a credit line to the material. If material is not included in the article's Creative Commons license and your intended use is not permitted by statutory regulation or exceeds the permitted use, you will need to obtain permission directly from the copyright holder. To view a copy of this license, visit http://creativecommons.org/ licenses/by/4.0/

(c) The Author(s) 2018 